\title{
Preemie Parent Perspective: Addressing Health Equity and Cultural Competency in the NICU
}

Jenné Johns, MPH

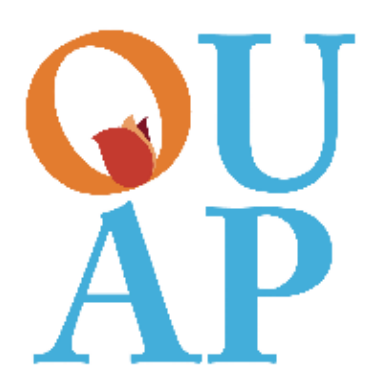

In 2016, I published Once Upon A Preemie, a first- of its kind children's book written to comfort parents of premature infants during their journey through the Neonatal Intensive Care Unit (NICU). During my journey, I discovered that reading to my micropreemie was the one activity as a mother that I could offer my son that helped normalize my overwhelming and traumatic NICU experience. During our nearly three-month stay in the NICU, I read to my son every day as research studies suggest that reading stimulates healthy brain development in preemies, and also helps to form a bond between parent and baby. Many of the bedtime stories that we read ended with a parent tucking the child into bed at home with Mommy and Daddy. That wasn't our reality for three months. There were no books about us.

Little did I know that in publishing my deepest emotions carried during and post NICU would lead me to become an author and speaker, but also an advocate and advisor for the needs of preemie parents, especially African Americans. As the mother of a micropreemie and miracle baby born at 26 weeks and weighing $1 \mathrm{lb} 15.3$ ounces, I found myself advocating for his needs as I knew his life depended on it. Despite my 10-year career working to eliminate racial and ethnic disparities in health care, nothing prepared me for the heart-wrenching experience of my son's premature birth. "Disparity" became real for me as my son joined the ranks of the nearly 500,000 premature babies born in the United States, nearly half to African American and Hispanic mothers. It was through this dual role that I experienced the NICU, one as a vulnerable micropreemie mother, and the other as a health equity professional.

\section{"My advocacy skills were tested daily, as his life depended on how well I could speak "neonatology" language, I had to be his voice and articulate his needs. This was challenging because, after all, “I'm just a Mom," an African American Mom, and not a doctor."}

At birth, my son required life-saving medical interventions; oxygen, phototherapy lights, feeding tubes, a heart monitor, medication, vitamins, and even caffeine. Over our nearly three-month stay in the NICU, I traveled through snowstorms and blizzards, to parent and nurture my baby. I only missed three days (two due to inclement weather and one self-care day). A typical day in the NICU lasted from 7 am until midnight, with many breaks to pump breastmilk. My lactation consultant promised that my breastmilk was liquid medicine. Midway through our NICU journey, I had to return to work, unlike many of my new NICU parent friends who were Caucasian. My advocacy skills were tested daily, as his life
Jenné Johns, MPH is the mother of a micropreemie, author, speaker, advocate, and national senior health equity leader. As an advocate for reducing healthcare disparities, Jenné found herself advocating for the needs of her son, as his survival depended on it. She also learned the power of reading to her son daily. Jenné wrote, "Once Upon a Preemie," which is the first of its kind children's book written for the parents of preemies while they are in the NICU. This bedside companion seeks to motivate, encourage, and inspire preemie babies and families until they go home.

Her preemie parent contributions include consulting and blogging for fortune 500 companies on preemie parent needs from a cultural lens and reading as a tool for growth, development, and bonding. She serves on numerous advisory committees advocating for preemie babies and eliminating health disparities, including NICU Parent Network, The National Coalition on Infant Health, and March of Dimes Prematurity Prevention Collaborative." She has presented at numerous conferences as a preemie parent, including Congressional Black Caucus Annual Legislative Conference, National Neonatal Nurses Association Conference, National Perinatal Social Workers Congressional Briefing, Preemie Parent Alliance, and National Perinatal Association Annual Conference. Jenné was featured in the Baby First Blog, Preemie World, Heart and Soul Magazine, iHeart Radio, CBS Philly News Radio, Disruptive Women in Healthcare Blog, and Women of a New Sisterhood.

In her professional capacity, Jenné is a National Senior Health Equity Thought Leader. Most recently, Jenné served as Director of Quality Improvement and Health Equity at Blue Cross and Blue Shield, Illinois, where she was responsible for leading strategies to improve health outcomes, reduce cost, and reduce racial and ethnic healthcare disparities with a focus on maternal and child health innovations. She also co-chaired the enterprise-wide Equity Steering Committee. Prior to Blue Cross and Blue Shield Illinois, Jenné served as Director of Health Disparities at AmeriHealth Caritas. She led innovations to reduce healthcare inequities for 5 million lives in 19 states in the areas of maternal and child health, pediatric asthma, diabetes, and cardiovascular disease. Jenné has over a decade of experience advocating for policy, business, and community changes to improve health outcomes for low-income communities through her work with Policy Link, Summit Health Institute for Research and Education, Robert Wood Johnson Foundation, and the National Nursing Centers Consortium.

Jenné is a graduate of the Disparities Leadership Program led Harvard Medical School. Her advisory appointments included: Pennsylvania Office of Health Equity (Advisor); Mid-Atlantic Regional Health Equity Council (Co-Chair); Regional Cancer Disparities Initiative at Thomas Jefferson Hospital (Co-Chair); Board Member, Pebbles of Hope. Her awards include Certificate of Recognition from the Honorable Congresswoman Robin Kelly (D-IL), Chair Congressional Black Caucus Health Braintrust; 40 Under Forty 2018 Achievement Award (The Network Journal); Top 100 Diverse Leaders Under 50 (Diversity MBA Magazine); Certificate of Recognition for the President's Commission on White House Fellowships Program (Regional Finalist); Certificate of Commendation-HHS Office of Minority Health, and Philadelphia Leader on the Move-Philadelphia Business Journal. 


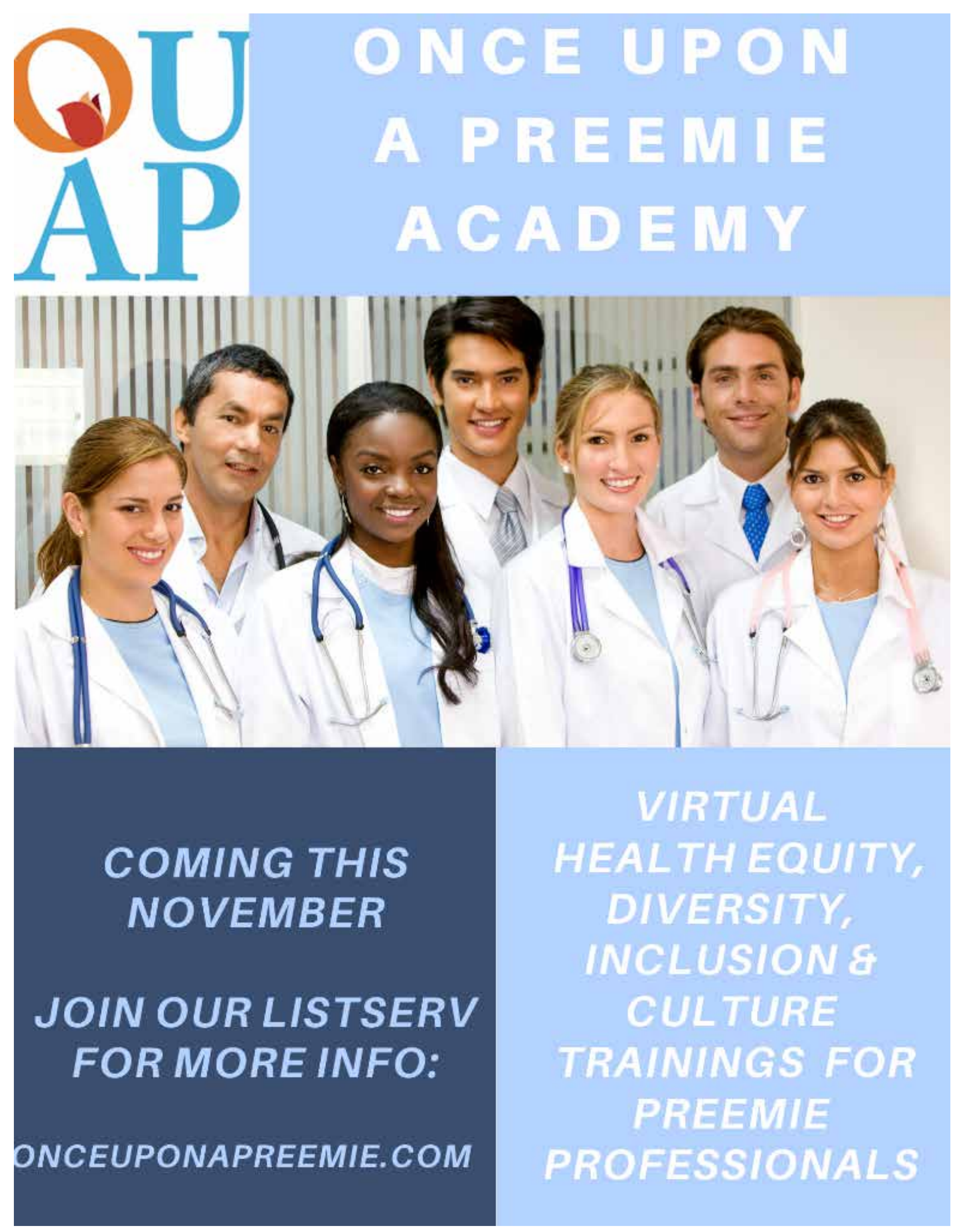


depended on how well I could speak "neonatology" language, I had to be his voice and articulate his needs. This was challenging because, after all, "I'm just a Mom," an African American Mom, and not a doctor.

\section{"I now believe, that with trauma-informed and implicit bias training among hospital staff, the professional staff would have been better equipped to communicate and support my delicate and fragile nature."}

As a mother, my NICU journey was traumatic and filled with a sea of emotions, including fear, anxiety, helplessness, and isolation. Much of which NICU parents are facing due to the current COVID-19 pandemic. Many of my fears, concerns, and feelings of isolation were due to the NICU environment, which was not as culturally friendly and supportive, as I assumed it would be. I'm being generous by saying there was little cultural diversity; it was dismal at best. There were times when the lack of cultural sensitivity and bedside manner caused more pain than my son's actual health status, and it made me very uncomfortable because as the end of each night, I had to trust my most prized possession with nurses and doctors who I did not always trust. Another challenge I faced as an African American preemie parent, was that although our larger hospital system had active and robust NICU parent support groups, these resources were not made available at the smaller hospital where I delivered my son. This hospital served more African American and lower-income families than the other hospitals. Many of the parents I developed a relationship with, felt as if our socio and emotional needs did not matter and were oftentimes dismissed because of this missing resource. Lastly, I experienced inconsistent positive communication and relationships with many of the NICU staff. Although I now believe that all of the members of my son's care team, held his safety and the quality of care they delivered to him with the utmost regard, our daily communication and interaction lacked humility, respect, and sensitivity. I will admit, I was not always the easiest or most cheerful mother to deal with, I now believe, that with trauma-informed and implicit bias training among hospital staff, the professional staff would have been better equipped to communicate and support my delicate and fragile nature.

Overall, a good deal of our NICU experience was positive; some experiences left permanent and negative memories that, to this day, cannot be erased. As much as I tried checking my professional credentials at the door before entering the NICU, my interactions with the NICU staff begged, yelled, and warranted us to have those tough cultural sensitivity conversations. Not in a negative way, but as an opportunity for forming better communication, respect, and, most importantly, trust.

In my professional view, the NICU is a microcosm of the larger hospital system on steroids, particularly NICU's serving low income and racially, ethnically, and linguistically diverse populations. Health disparities impacting the NICU are also a reflection of a larger hospital ecosystem. Below are my preemie parent and professional recommendations for integrating health equity and cultural competency in the NICU:

1. Prioritize health equity and cultural competency as strategic priorities and goals. Establishing opportunities for integrating and addressing health equity in short and long terms strate- gies ensures layers of accountability, allocation of funding, measurement, and documentation of outcomes. One example of an important health equity priority includes staff diversity. Peer-reviewed studies have shown that cultural congruence among patients and providers yields better health outcomes, better communication, and trust.

2. Make health equity, cultural competency, and implicit bias training mandatory for all NICU Staff. Participating in an annual training program is a great start to begin addressing and delivering equitable care to all NICU families. However, one-time training is not sufficient. Integrating health equity and implicit bias content into clinical rounds, staff development, and training opportunities are critical to reducing racial and ethnic disparities in the NICU.

3. Communicating in lay terms should be standard in every NICU. Literacy and health literacy levels are important considerations for family-centered and culturally appropriate care in the NICU. Regardless of one's educational level, the NICU terminology is overwhelming and confusing for a new parent entering the NICU. Literacy and health literacy considerations are also important factors for families who are limited or non-English speaking. Break the communication barriers by speaking the same language and utilizing interpreters even if everyone speaks English. I had a great deal of respect and appreciation for the NICU staff who used lay terms and avoided NICU jargon when communicating with me. In time, I began understanding the NICU language; however, that wasn't my job as a preemie parent. Preemie parents should be made to feel as comfortable speaking and interacting with NICU staff regardless of their literacy and health literacy levels.

4. Partner with parents to address the cultural competency, spiritual diversity, and unconscious biases that exist in the NICU. Listen to the voices of parents with multicultural backgrounds to be more sensitive to racial, ethnic, language, income, education, transportation, and spiritual needs. Encourage preemie parents to speak up. Staff should value their input. Allow parents to give their insights on their baby's health status, and any gut feelings they may have about a diagnosis or new development. This is extremely important for minority parents who assume their voice and parental role is undervalued.

5. Engage and establish culturally congruent NICU family supports. Many minority parents may not immediately express a need for mental or emotional help while in the NICU for fear of being labeled. Where and when possible, make culturally congruent resources available to support these parents, even if the supports are outside of the NICU.

6. Make digital technology and virtual solutions available to parents with transportation, competing work schedules, or other barriers to delivering care to their preemies. This is most critical during the current COVID season, where parental fears and social distancing may prohibit them from

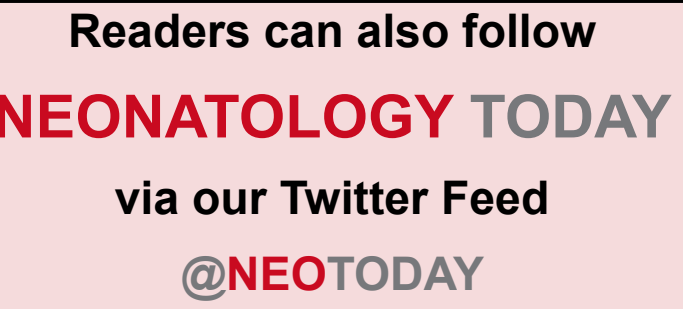


visiting their baby. Creating safe opportunities for parents to connect with their babies is vital bonding via smart devices or other safe technology solutions.

"This November, as we go purple in recognition of Prematurity Awareness Month, we hope that you will join us as we launch the Once Upon A Preemie Academy, a virtual health equity and cultural competency training program for preemie professionals and parents."

This November, as we go purple in recognition of Prematurity Awareness Month, we hope that you will join us as we launch the Once Upon A Preemie Academy, a virtual health equity and cultural competency training program for preemie professionals and parents. For more information about the Once Upon A Preemie Academy and for additional health equity and cultural competency resources, please join our listserv and visit these online resources:

1. Once Upon A Preemie Academy Listserv: www.onceuponapreemie.com

2. BabyFirst Blog Post: Culture Matters in the NICU https:// www.babyfirst.com/en/blog-posts-jenne-johns/culture-matters-in-the-nicul

3. BabyFirst Blog Post: The Importance of Reading to your Preemie. https://www.babyfirst.com/en/blog-posts-jennejohns/the-importance-of-reading-to-your-preemie/

Disclosure: The author has no disclosures.

NT

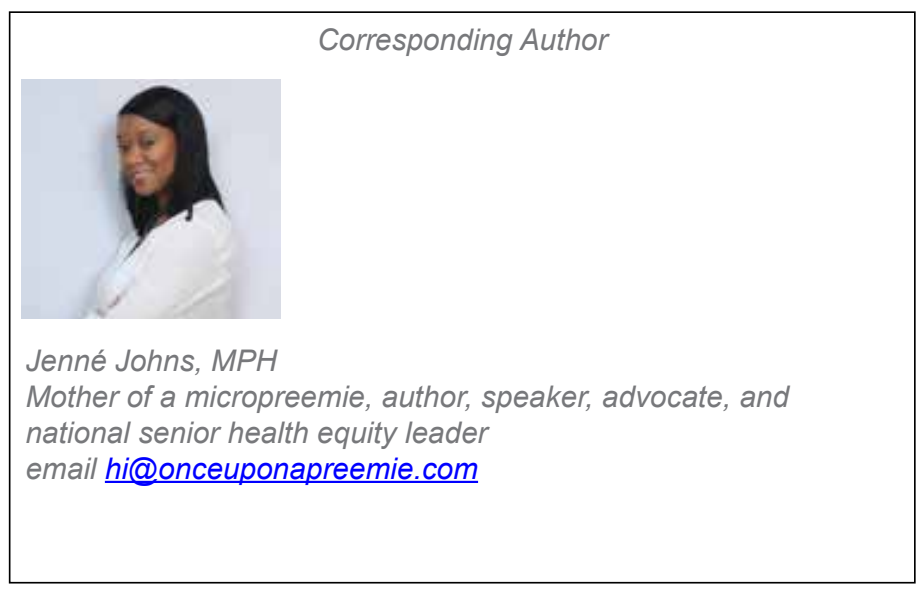

\section{Why PREMATURE INFANTS Need Access \\ to an EXCLUSIVE HUMAN MILK DIET}

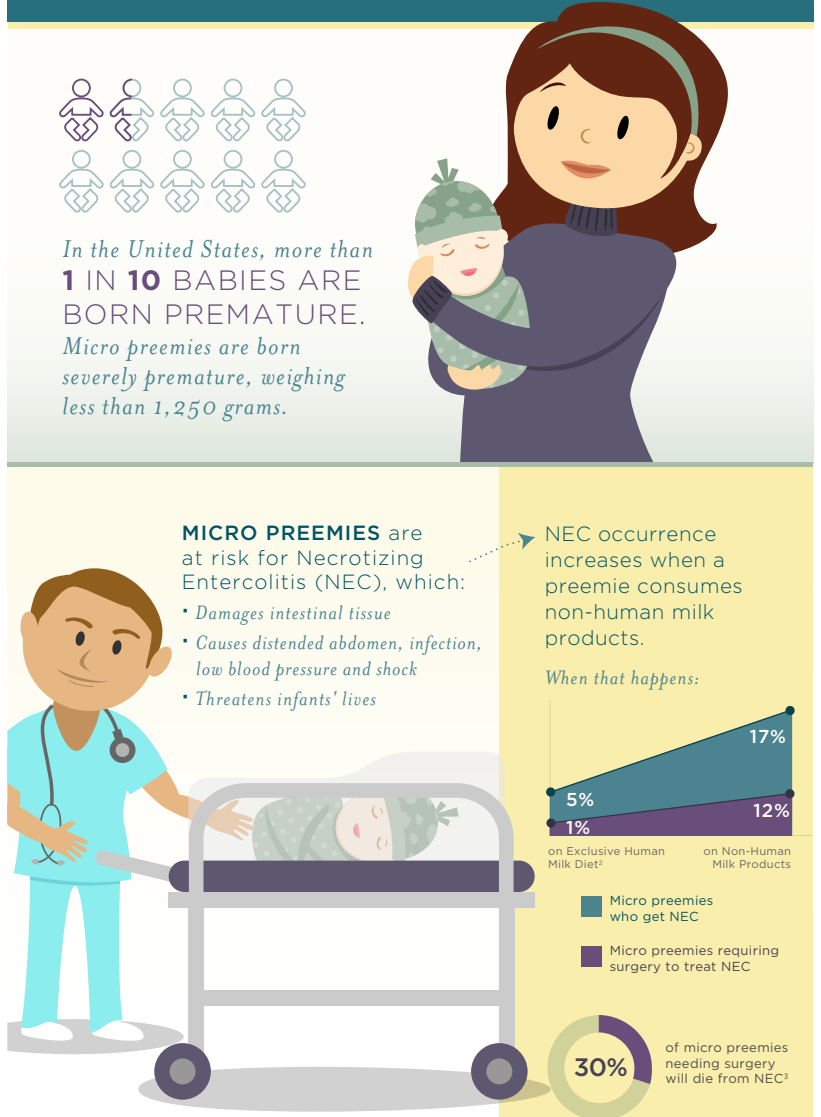

HOW TO HELP PREVENT NEC: EXCLUSIVE HUMAN MILK DIET

What is an Exclusive Human Milk Diet?
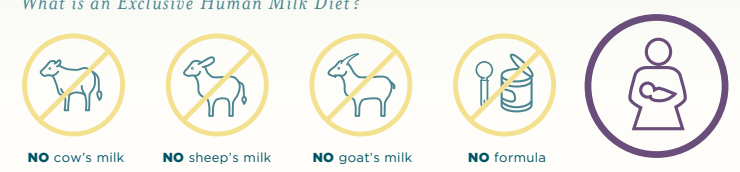
$\checkmark$ mother's milk $\checkmark$ human donor milk

Why Is An Exclusive Human Milk Diet Important?

An Exclusive Human Milk Diet gives vulnerable infants the best chance

to be healthy and reduces the risk of NEC and other complications.

When a micro preemie can access an EXCLUSIVE HUMAN MILK DIET:
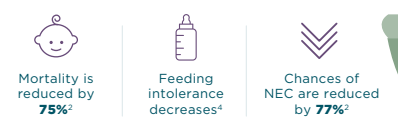

HUMAN MILK = MEDICINE

LEARN MORE •

NCfIH

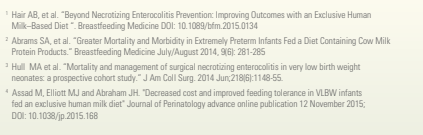

